\title{
Oxygen derived free radicals in patients with chronic pancreatic and other digestive diseases
}

\author{
D Basso, M P Panozzo, C Fabris, G del Favero, T Meggiato, P Fogar, A Meani, \\ D Faggian, M Plebani, A Burlina, R Naccarato
}

\begin{abstract}
To ascertain modifications in the activation products derived from oxygen free radicals in patients with chronic pancreatic and extra-pancreatic diseases, lipid peroxide activity was measured in the sera of 40 control subjects, 28 patients with pancreatic cancer, 49 with chronic pancreatitis, and 53 with extra-pancreatic diseases. In 142 of the subjects, elastase 1, amylase, and pancreatic isoamylase activities were also determined. Increased lipid peroxide activities were found in some patients with both chronic pancreatic and extra-pancreatic diseases. Patients with chronic pancreatitis studied during relapse had higher activities of lipid peroxides than those without active disease. No difference was found between the values in patients with pancreatic cancer with liver metastases and those without. Correlations were found between lipid peroxides and both amylase and pancreatic isoamylase activities; no correlation was detected between lipid peroxides and elastase 1 . In benign biliary tract disease a correlation was detected between lipid peroxides and alanine aminotransferase and alkaline phosphatase activities. In all patients, however, a correlation was found between alkaline phosphatase and lipid peroxide activities.

It is concluded that activation of oxygen derived free radicals occurs in chronic pancreatic as well as in extra-pancreatic disease; it seems to reflect the degree of inflammation.
\end{abstract}

Istituto di Medicina Interna (Cattedra di Malattie Apparato Digerente) Padua D Basso

M P Panozzo

C Fabris

G del Favero

T Meggiato

P Fogar

A Meani

$R$ Naccarato

Cattedra di Chimica Microscopia Clinica, Università degli Studi di Padova, Italy

D Faggian

M Plebani

A Burlina

Correspondence to:

Professor Remo Naccarato,

Istituto di Medicina Interna, Cattedra di Malattie Apparato Digerente, Policlinico Universitario, Via Giustiniani 2, 35100 Padova Italy

Accepted for publication 21 December 1989 of acute pancreatitis. ${ }^{2}$ Both enzymes play a part in the metabolism and scavenging of oxygen derived free radicals; their administration should prevent the accumulation of free radicals in tissues.
An increase in mixed function oxidasesenzymes that are involved in the hepatic metabolism of toxic substances, and which produce free radicals-has been found in patients with chronic pancreatitis. ${ }^{7}$ It has therefore been suggested that the activation of oxygen derived free radical products might have a role in the pathogenesis of chronic pancreatitis. ${ }^{89}$

All the above studies have indicated that oxygen derived free radicals may be involved in the pathogenesis of pancreatic diseases, but to date there are no studies on the amount of free radicals activated during the course of different chronic pancreatic diseases.

We investigated serum variations in the activation products of oxygen derived free radicals such as lipid peroxides in patients with chronic pancreatic diseases and compared them with values obtained in the sera of patients with other gastrointestinal diseases. We also wanted to determine whether there was any association between free radical activity and pancreatic damage or other pathological findings.

\section{Methods}

One hundred and seventy subjects were studied: the control group comprised 40 (29 men, 11 women, age range 19-66 years), either healthy members of the medical staff or blood donors. Twenty eight patients had pancreatic cancer of duct cell origin (12 men, 16 women, age range 28-76 years), which had been histologically confirmed ${ }^{10}$ in intraoperative or necropsy specimens; 16 had liver metastases. Forty nine had chronic pancreatitis (45 men, four women, age range 27-83 years), diagnosed on the basis of the clinical picture and on the positive findings for at least two of the following: abdomen $x$-ray pictures for pancreatic calcifications, ultrasonography, computed tomography, endoscopic retrograde pancreatography: 19 patients were studied during a relapse. Fifty three had extra-pancreatic diseases ( 32 men, 21 women, age range 35-78 years), diagnosed on the basis of the clinical picture and appropriate radiological or histological findings or a combination. Of these, eight had extra-pancreatic malignancies (primary liver cell cancer $\mathbf{n}=3$; duodenal lymphoma $n=1$; colorectal carcinoma $n=1$; carcinoma of the gallbladder $n=1$; carcinoma of the hepatic hilus $n=1$; carcinoma of the thyroid gland $\mathbf{n}=1$ ). Twenty had benign liver diseases (liver cirrhosis $n=14$; liver steatofibrosis $\mathrm{n}=2$; liver angioma $\mathrm{n}=2$; chronic hepatitis $n=2$ ). Fifteen had benign biliary tract diseases (gallstones $n=7$; benign stenosis of the papilla of Vater $n=3$; cholan- 
Figure 1 Individual values of serum lipid peroxide concentrations. Continuous line represents (mean + $2 S D$ of our controls). O: chronic pancreatitis studied during a relapse; $\boldsymbol{\Delta}$ : metastatic pancreatic cancers. the upper normal limit

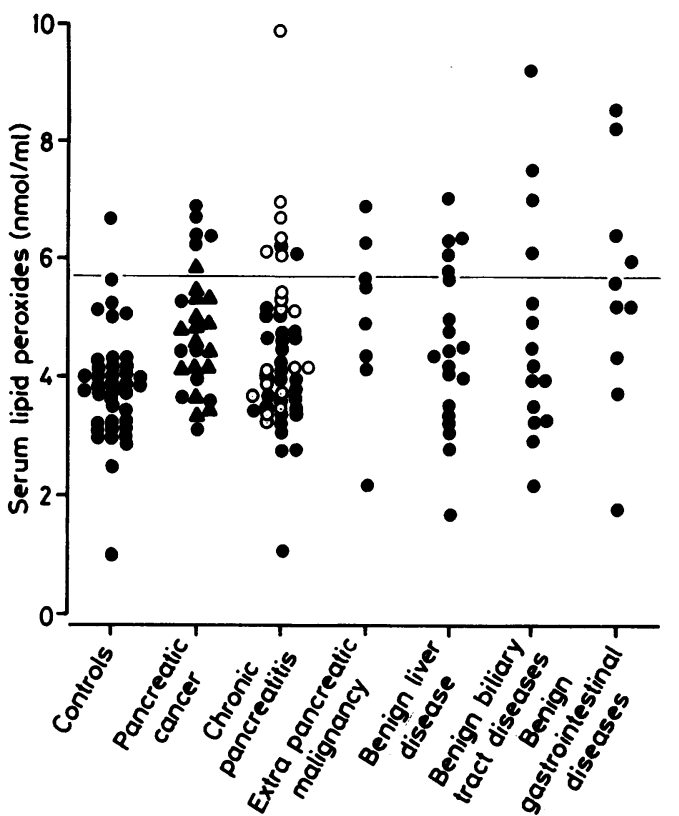

gitis $\mathrm{n}=2$; choledocholithiasis $\mathrm{n}=1$; empyema of the gallbladder $\mathrm{n}=1$; sclerosing cholangitis $\mathrm{n}=1$ ). Ten had benign gastrointestinal disease (duodenal ulcer $n=3$; irritable colon $\mathrm{n}=2$; Crohn's disease $\mathrm{n}=2$; coeliac disease $\mathrm{n}=1$; diverticulosis of the colon $\mathrm{n}=1$; hiatus hernia $\mathrm{n}=1$ ).

Serum lipid peroxides were measured according to Yagi's method, ${ }^{11}$ which fluorimetrically determines the reaction occurring between lipid peroxidation products and thiobarbituric acid (TBA). In 142 subjects elastase 1 and total and pancreatic isoamylase were assayed by the following procedures: radioimmunoassay technique (Abbott); Rauscher's method ${ }^{12}$; and pancreas specific monoclonal antibody assay. ${ }^{13}$

Data were evaluated by analysis of variance (Anova one way), Bonferroni's test for pairwise comparisons, ${ }^{14}$ and Student's $t$ test.

\section{Results}

Figure 1 specifies the individual lipid peroxide values in the material studied; the table reports the mean (SEM) values and the statistical analysis of lipid peroxide concentrations.

Figure 2 shows the results for the three pancreatic enzymes. No significant differences were detected between the lipid peroxide concentrations of patients with pancreatic cancer with metastases and those without liver $(t=$ $1 \cdot 34, \mathrm{p}=\mathrm{NS})$. Patients with chronic pancreatitis studied during a relapse had higher lipid peroxide concentrations than those without active disease $(t=2.86, \mathrm{p}<0.01)$. In these

Serum lipid peroxidation products

\begin{tabular}{llll}
\hline & \multicolumn{3}{c}{ Lipid peroxidation products $($ nmol $/ \mathrm{ml})$} \\
\cline { 2 - 4 } & No & Mean & SEM \\
\hline Control subjects & 40 & $3 \cdot 85$ & $0 \cdot 15$ \\
Pancreatic cancer & 28 & $4 \cdot 81^{\star}$ & $0 \cdot 20$ \\
Chronic pancreatitis & 49 & $4 \cdot 43^{\star}$ & $0 \cdot 20$ \\
Extra-pancreatic malignancy & 8 & $5 \cdot 00$ & $0 \cdot 52$ \\
Benign liver diseases & 20 & $4 \cdot 53$ & $0 \cdot 31$ \\
Benign biliary tract diseases & 15 & $4 \cdot 81^{\star}$ & $0 \cdot 50$ \\
Benign gastrointestinal diseases & 10 & $5 \cdot 51^{\star}$ & $0 \cdot 63$ \\
Total & 170 & & \\
\hline
\end{tabular}

Analysis of variance $f=3.06, p<0.01$.

Bonferroni's test ${ }^{\star} p<0.01$ compared with control subjects. patients a negative correlation was found between lipid peroxides and the white cell count $(\mathrm{r}=-0.4218, \mathrm{p}<0.01)$.

When the patients were considered together, significant correlations were found between lipid peroxide values and amylase $(r=0.3314$, $\mathrm{p}<0.001)$ and between lipid peroxides and pancreatic isoamylase $(r=0.2757, p<0.05)$. No correlation was found between lipid peroxide and elastase $1(r=0 \cdot 1248, p=N S)$.

Positive correlations were also found between the reciprocal transformation of creatinine values and lipid peroxides in patients with pancreatic cancer $(r=-0.5333$, $\mathrm{p}<0.01$ ), with benign biliary tract diseases $(\mathrm{r}=-0.9095, \mathrm{p}<0.01)$, and with gastrointestinal disease $(\mathrm{r}=-0.7642$, $\mathrm{p}<0.05)$. In patients with benign biliary tract disease a relation was found between lipid peroxides and alanine-aminotransferase $(\mathrm{ALT})(\mathrm{r}=0.6911, \mathrm{p}<0.01)$ and with alkaline phosphatase $(\mathrm{r}=0.5412, \mathrm{p}<0.05)$. In all patients a correlation was found between alkaline phosphatase activity (ALP) and lipid peroxide concentration $(r=0.3745$, $\mathrm{p}<0.001)$.

\section{Discussion}

Lipid peroxide concentrations were measured in our subjects to quantitate indirectly the activation products of oxygen derived free radicals. Lipid peroxides were found in healthy subjects, suggesting that oxygen free radicals are also produced in the normal state, probably as a consequence of normal metabolic and catabolic processes.

Increased lipid peroxide concentrations were found in some patients with both chronic pancreatic or extra-pancreatic diseases, indicating that there is a non-specific overproduction of free radicals in some phases of the disease. In our patients, however, we did not observe pronounced variations in lipid peroxide concentrations, probably because of their clearance and the dilutional effect of circulation.

Our study suggests that there is an obvious association between the inflammatory state of various organs during different diseases and the amount of lipid peroxides detected. This applies to both chronic pancreatic and extrapancreatic diseases. In chronic pancreatitis higher lipid peroxide values were found in patients with active inflammation, as evidenced by biochemical and clinical investigations. In patients with chronic pancreatic diseases correlations were found between lipid peroxide concentrations and amylase and isoamylase activities. In patients with pancreatic cancer lipid peroxide values showed no variations, even if the tumour was metastatic. This suggests that in pancreatic neoplasia peritumoral cell inflammation has an important role in increasing serum lipid peroxide concentrations. This is compatible with our failure to find any correlation with elastase activity. The latter does not present the same variations as amylase, depending, at least in part, on different pathophysiological mechanisms. Sur- 
Figure 2 Individual values of serum elastase 1 , amylase, and pancreatic isoamylase arthritis. Continuous lines represent upper limits of normal. $O$. chronic pancreatitis studied during a relapse: $\Delta$ : metastatic pancreatic cancers.

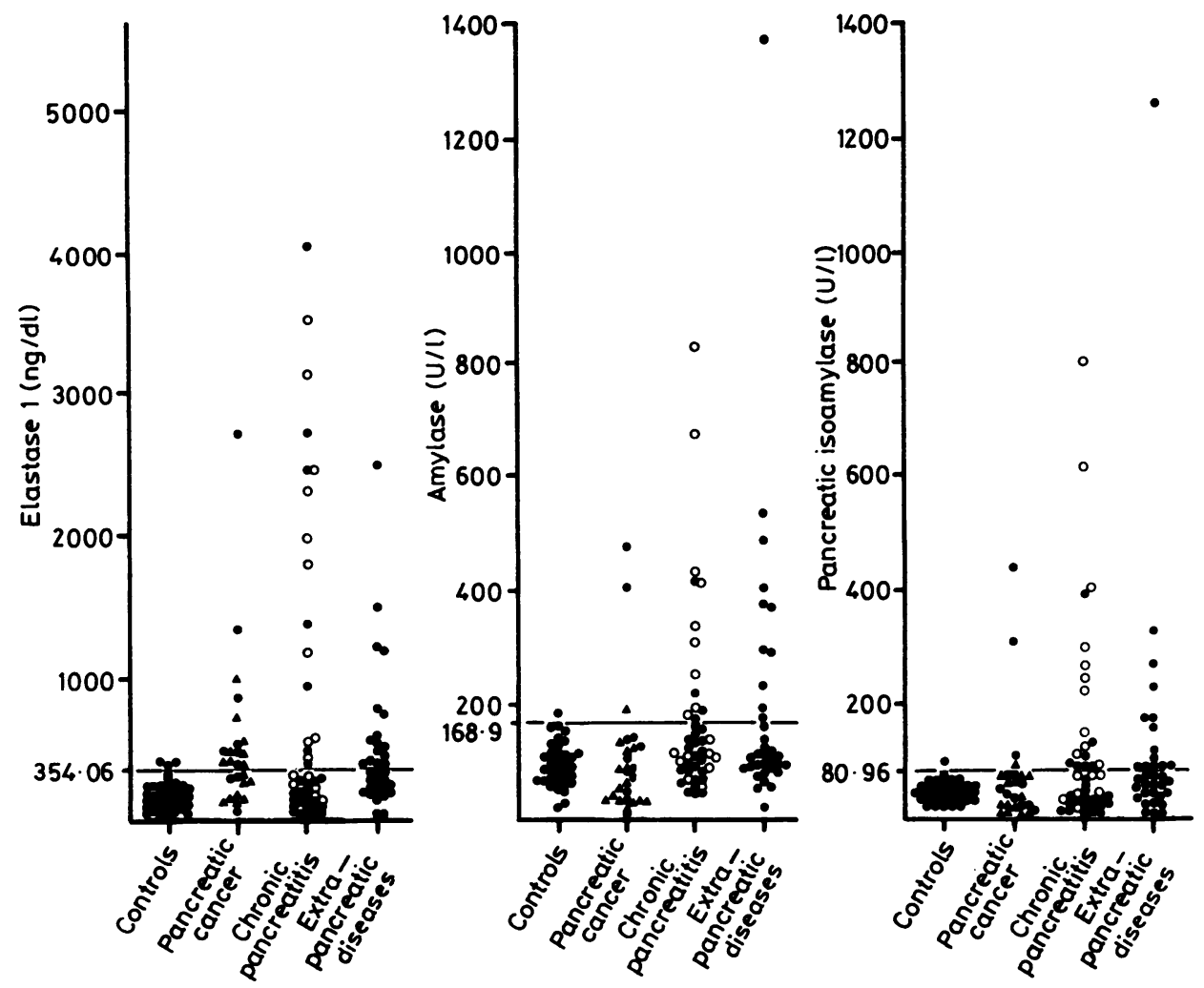

tion. The exact role of this process in such

prisingly, we detected an inverse correlation in chronic pancreatitis between the white cell count and activation products of oxygen derived free radicals. Free radicals are not produced exclusively by leucocytes. In chronic pancreatitis, a disease in which cell and tissue necrosis starts at the gland itself without affecting the white cell count, the fluctuation in lipid peroxide concentrations may depend on pancreatic glandular damage.

In biliary tract disease we found that lipid peroxide concentrations were correlated with ALT and ALP activities, which, in these diseases, reflect the activity and severity of the inflammation. The correlation is therefore not surprising. Furthermore, the correlation found between ALP and lipid peroxides in all patients is easily explained because ALP is ubiquitous and increases in several types of inflammation in the same way that lipid peroxides do.

Finally, in some groups of patients (those with pancreatic cancer, benign biliary tract disease, and gastrointestinal disease) correlations were found between serum creatinine and lipid peroxide concentrations. Recent studies have emphasised the crucial role of oxygen derived free radicals in the pathogenesis of renal dysfunction and failure, these substances being mediators of post-ischaemic injury to the kidney. ${ }^{15}$ Various degrees of renal damage have been described in pancreatic cancer and biliary tract diseases ${ }^{16}$ because in these conditions kidney damage may be proportional to the degree of pancreatic inflammation, to hyperbilirubinaemia, and to the severity of other pathological factors. In our patients, therefore, activation of oxygen derived free radicals may have played a part in determining renal disease.

In conclusion, we found that the activation of oxygen derived free radicals occurs in chronic pancreatic as well as in extra-pancreatic diseases, where it reflects the degree of inflamma- patients, however, is not well understood.

This work was supported in part by a grant from Centro Regionale di Alta Specializzazione per lo Studio delle Malattie del Fegato e del Pancreas and carried out under the auspices of the $\mathbf{R}$ Farini association for gastroenterological research.

1 Rutledge PL, Saluja AK, Powers RE, Steer ML. Role of oxygen-derived free radicals in diet-induced hemorrhagic
pancreatitis in mice. Gastroenterology 1987;93:41-7.

pancreatitis in mice. Gastroenterology 1987;93:41-7.
2 Wisner J, Green D, Ferrell L, Renner I. Evidence for a role of oxygen derived free radicals in the pathogenesis of caerulein induced acute pancreatitis in rats. Gut 1988;29:1516-23.

3 Sarr MG, Bulkley GB, Cameron JI. The role of leukocytes in the production of oxygen-derived free radicals in acute experimental pancreatitis. Surgery 1987;101:292-6.

4 Southorn PA, Powis G. Free radicals in medicine. I. Chemical nature and biologic reactions. Mayo Clin Proc 1988;63:381-9.

5 Southorn PA, Powis G. Free radicals in medicine. II. Involvement in human disease. Mayo Clin Proc 1988; Involvement

6 Sava P, Blondeau C, Cubertafond P, Magnin P. Radicaux libres et pathologie digestive. Gastroenterol Clin Bio $1988 ; 12: 214-21$

7 Braganza JM, Wickens DG, Cawood P, Dormandy TL. Lipid peroxidation (free-radical-oxidation) products in bile from patients with pancreatic disease. Lance 1983;ii:375-9.

8 Braganza JM. Pancreatic disease: a casualty of hepatic "detoxification"? Lancet 1983;ii:1000-3.

9 Acheson DWK, Rose P, Houston JB, Braganza JM Induction of cytochromes P-450 in pancreatic disease: Induction of cytochromes $\mathrm{P}-450$ in pancreatic disease: consequence, $1985 ; 153: 73-84$.

10 Cubilla AL, Fitzgerald PJ. Pancreas cancer. 1. Duct adenocarcinoma. Pathol Annu 1978;13:241-89.

11 Yagi K. Assay for blood plasma or serum. Methods in Enzymology 1984;105:328-31.

12 Rauscher E, Neumann U, Schaich E, Von Bulow S, Wahlefeld AW. Optimised conditions for determinin 4-nitrophenylmaltoheptaoside as substrate. Clin Chem 1985;31:14-9.

13 Gerber $M$, Naujoks $K$, Lenz $H$, Wulff K. A monoclonal antibody that specifically inhibits human salivaryamylase. Clin Chem 1987;33:1158-62.

14 Wallenstein S, Zucker CL, Fleiss JL. Some statistical methods useful in circulation research. Circ Res 1980;47:1-9.

15 Canavese C, Stratte P, Vercellone A. The case for oxygen free radicals in the pathogenesis of ischemic acute renal failure Nephron 1988;49.9-15.

16 Fabris C, Basso D, Del Favero G, et al. Renal tubular dysfunction in pancreatic cancer and chronic pancreatitis. Nephron 1989;51:56-60. activity concentration of -amylase in serum, with 1,4- -D- 